\title{
Tumor genotyping for breast cancer: at the front door
}

\section{"One emerging solution is the development of biology-driven clinical trials, in which candidate patients are those that harbor the molecular alteration targeted by the drug."}

\section{KEYWORDS: breast cancer $\approx$ high-throughput technologies $\approx$ molecular screening - personalized medicine $\approx$ targeted therapy}

\section{Why run high-throughput technologies in metastatic breast cancer patients?}

Historically, the classification of breast cancer has been made based on the presence and/or overexpression of standard biomarkers such as estrogen receptor (ER), progesterone receptor (PR) and HER2. Tumors have been classified as hormone-receptor positive and/or HER2 ${ }^{+}$and in triple-negative breast cancer as the absence of these three markers.

Treatment decisions are usually made based on these subgroups divisions in addition to other cliniopathological features.

"...the current goal of the molecular screening program should be to speed up drug development (logistical end point), but not (yet) survival improvement."

During the recent years, owing to the introduction of high-throughput technologies there has been an increased number of newly discovered oncogenic molecular alterations. The majority of such relevant oncogenic molecular alterations occurs only in a small percentage of patients and defines a specific segment of the disease [1-5]. Moreover, some of these molecular alterations may coexist and define different phenotypes such as, for example, ERBB2 amplification and PIK3CA mutations that might be associated to trastuzumab resistance [6].

Most of the new drugs under early development target molecular alterations in breast cancer but when these drugs are tested in Phase I and Phase II trials, there is no selection of patients with this specific alteration; therefore there is the risk to falsely conclude that a new agent does not work if the trial does not include a minimum number of patients.
Initial strategies in order to discover predictors of response or benefit from a targeted therapy were based on retrospective biomarker analyses of Phase II/III trials, which has allowed the identification of several biomarkers, such as $K$-ras mutations to predict cetuximab resistance [7] or response to anti-EGFR treatment in lung tumors harboring EGFR mutations [8]. Although this strategy has been proven to be successful in these cases, is not an appropriate strategy in rare oncogenic events essentially because it would require larger series of patients to retrospectively address this biomarker hypothesis.

One emerging solution is the development of biology-driven clinical trials, in which candidate patients are those that harbor the molecular alteration targeted by the drug.

The most commonly used scenario is the one that implies the test of a single molecular alteration, where the assay is performed after the patient has signed the informed consent for the Phase II trial. Several restrictions are associated to this model: screening failure is quite high, especially in rare or low frequent alterations and patients are currently in progression of their disease after certain number of lines of treatment. Therefore, if patients are not eligible for this trial it is quite unlikely they could be included in a study that involves a second molecular screening since treatment delays may not be acceptable.

A second scenario that would counteract the main problems associated with the trial design described above, would include patients at any time during the course of the disease without any direct link to a specific clinical trial and would assess a limited number of previously described molecular biomarkers. If any of the tested molecular alterations are present, then patients will be eventually eligible for a specific

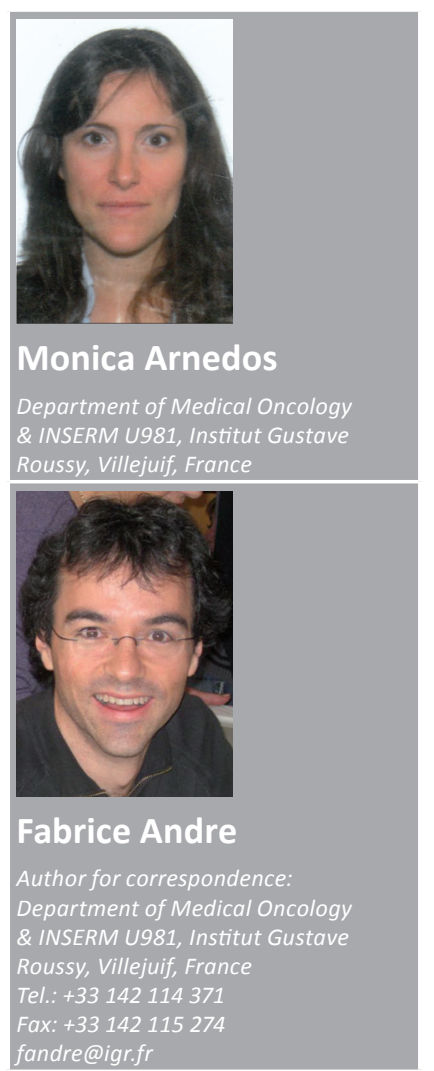

future medictio $^{\text {pisg }}$ fs 
clinical trial targeting that molecular alteration. However, a major drawback is the fact that the tested molecular alterations are those that are well known, so therefore limited. And as new relevant genomic alterations become available, this will require the development of new bioassays to identify them.

\section{High-throughput technologies for routine molecular screening in Phase I/II trials}

Taking into account the main limitations present with the two previously mentioned approaches, a third trial design proposal that represents a better proposal would be one that performs the molecular testing at any time, and it is not linked to any specific Phase II trial in terms of main consent. The main difference with the previous scenario would be the fact that molecular screening will be performed using high-throughput technologies instead of a dedicated bioassay for each molecular alteration. These high-throughput technologies include microarray gene-expression profiling that quantifies genome-wide gene expression and comparative genomic hybridization (CGH) array analysis that quantifies DNA copy number variation.

"...the major caveat associated with performing molecular profiling is the risk of excess information and what we do with it."

An example of this proposal is the SAFIR01 trial sponsored by the French National Cancer Institute and is due to start recruitment. In this French cooperative study, patients with advanced breast cancer during the course of their treatment, and without evidence of progressive disease, consent for a frozen biopsy of a metastatic site. Array GCH and hotspot mutations of PIK3CA and AKT will be performed in three different centers in France using different platforms. The primary end point is the percentage of patients included in Phase I/II trials according to their molecular profile, not the outcome's improvement. Indeed, the current goal of the molecular screening program should be to speed up drug development (logistical end point), but not (yet) survival improvement.

Major advantages associated to this model includes; rare but potentially relevant genomic events could be identified; high-throughput technologies enable the detection of multiple genomic events in the same sample; therefore a patient can be allocated to a trial according to the molecular alterations that present and this will enrich Phase I/II trials with a population of patients harboring the targeted alterations.

Nonetheless, this scenario also has some limitations. The first obvious one, apart from the costs derived from the realization of highthroughput technologies, is the feasibility of performing these techniques in regular daily practice. In our center, we have conducted a pilot study including more that 100 patients with metastatic breast cancer and we have found that using a dedicated biopsy team to obtain a frozen sample we could perform CGH arrays and hotspot mutation analysis in a significant majority of the cases. Moreover, we observed a good correlation between our results from gene amplification by fluorescence in situ hybridization and protein expression by immunohistochemistry for standard biomarkers, that is, HER2. Interestingly, approximately $20 \%$ patients were included in a specific clinical trial based on the molecular results.

In our opinion, the major caveat associated with performing molecular profiling is the risk of excess information and what we do with it. In fact, the main issue is not only to identify which mechanisms or molecular events are altered in a specific case, but also to distinguish which of them are relevant, if there are any that may be linked between them and specifically how to functionally address the results obtained from these molecular analysis. Only when we will be able to know these two elements, we will then achieve the major goal with the introduction of high-throughput technologies in standard daily practice, which is ultimately to improve the patients' outcome.

In conclusion, the use of high-throughput technologies could allow enrichment of Phase I/II clinical trials in patients who present a candidate molecular alteration. For logistical reasons, such molecular screenings should be carried out in patients who do not present a progressive disease. Also, such prospective programs should test 'logistical' end points instead of 'outcome' related end points. These molecular screening programs will be fine-tuned in the next years by the emergence of functional assays that test the activation of candidate pathways. Finally, the ability to integrate the genomic and the functional information should allow the generation and the test of the medical use of predictive algorithms. Next-generation trials will very likely focus on these algorithms and no longer on the drugs themselves. 


\section{Financial \& competing interests disclosure}

The authors have no relevant affiliations or financial involvement with any organization or entity with a financial interest in or financial conflict with the subject matter or materials discussed in the manuscript. This includes employment, consultancies, honoraria, stock ownership or options, expert testimony, grants or patents received or pending, or royalties.

No writing assistance was utilized in the production of

this manuscript.

\section{Bibliography}

1 Vogel CL, Cobleigh MA, Tripathy D et al. Efficacy and safety of trastuzumab as a single agent in first-line treatment of HER2overexpressing metastatic breast cancer. J. Clin. Oncol. 20, 719-726 (2002).

2 Mok TS, Wu YL, Thongprasert $S$ et al. Gefitinib or carboplatin-paclitaxel in pulmonary adenocarcinoma. N. Engl. J. Med. 361, 947-957 (2009).

3 Soda M, Choi YL, Enomoto $\mathrm{M}$ et al. Identification of the transforming EML4-ALK fusion gene in non-small-cell lung cancer. Nature 448, 561-566 (2007).
4 Shaw AT, Yeap BY, Mino-Kenudson M et al. Clinical features and outcome of patients with non-small-cell lung cancer who harbor EML4-ALK. J. Clin. Oncol. 27, 4247-4253 (2009).

5 Turner N, Lambros MB, Horlings HM et al. Integrative molecular profiling of triple negative breast cancers identifies amplicon drivers and potential therapeutic targets. Oncogene 29, 2013-2023 (2010).

6 Berns K, Horlings HM, Hennessy BT et al. A functional genetic approach identifies the PI3K pathway as a major determinant of trastuzumab resistance in breast cancer. Cancer Cell 12, 395-402 (2007).
7 Lievre A, Bachet JB, Le Corre D et al. KRAS mutation status is predictive of response to cetuximab therapy in colorectal cancer. Cancer Res. 66, 3992-3995 (2006).

8 Shih JY, Gow CH, Yang PC. EGFR mutation conferring primary resistance to gefitinib in non-small-cell lung cancer. $N$. Engl. J. Med. 353, 207-208 (2005). 